\title{
Beam Spreading and Spatial Resolution in Thick Organic Specimens
}

\author{
J.K. Hyun, ${ }^{*}$ P. Ercius, ${ }^{* *}$ and D.A. Muller** \\ * Department of Physics, Cornell University, Ithaca, NY 14853 \\ ** Department of Applied and Engineering Physics, Cornell University, Ithaca, NY 14853
}

As there are no post-specimen imaging lenses in the scanning transmission electron microscope (STEM) and hence no additional chromatic blurring from energy losses in the sample, STEM offers an intriguing potential for the 3D imaging of thick biological specimens where the image resolution is potentially limited only by beam spreading in the sample. This involves a good understanding of the relationship between thickness and spatial resolution. Earlier measurements by Beorchia et al [1] illustrate this relation for amorphous carbon substrates, but with the advances in brightness that easily accessible FEG electron sources offer, a proper analysis of the problem with particular emphasis on the competition between beam divergence and beam spreading is essential.

In general, features in a thick sample are blurred due to contributions from beam divergence and beam spreading effects. Beam spreading results from scattering events within the sample and is inherent to the nature of the sample. However beam divergence originates from geometrical optics, and the dominance of one effect over the other at the thickness of interest can be controlled by choosing the proper aperture size as shown in Fig. $1 \mathrm{a}$.

Beam spreading and beam divergence in an amorphous carbon substrate of varying thicknesses were studied with a 200kV FEI TECNAI TF-20 STEM system in annular dark field using a 9 mrad aperture (the optimal aperture for our spherical aberration coefficient of $1.2 \mathrm{~mm}$ ) [2]. A $100 \mathrm{~mm}$ camera length is used for all measurements. Samples are prepared by microtoming epon films up to $1 \mu \mathrm{m}$ in thickness, depositing $2 \mathrm{~nm}$ Au particles on top and bottom of the film, and carbon coating up to $15 \mathrm{~nm}$ to reduce radiation damage.

Beam divergence is determined by focusing on the bottom particles, measuring the blurring of the top particles and correcting for the finite particle size (Fig 1b). Beam spreading is obtained similarly but by measuring the width of the in-focus image of the bottom particles. Focusing on the top film and measuring the spread of the bottom particles shows the contribution from both spreading and divergence (Fig. 1c). When focused on the bottom of the film, the top particles blur into donut-like shapes for a thick substrate as shown in Fig. 2a. A quantum mechanical calculation of the probe shape is shown in Fig. $2 b$ and generates concentric rings at higher defocus values responsible for the donut-shaped blurring. In very thick samples, beam spreading determines the resolution limit but at intermediate $(\sim 100$ $\mathrm{nm}$ ) thicknesses it is the depth of field. There the resolution can be optimized by balancing beam divergence against the diffraction limit [4]. 
References

[1] A. Beorchia et al.,J. Microsc. 170 (1992) 247.

[2] M. Weyland, D.A. Muller. Nano Sol. 1 (2005) 25.

[3] H. Rose, $9^{\text {th }}$. Int. Con. Elec. Microsc. 3 (1978) 230.

[4] This work is supported by NSF (DMR-0405195, facilities:MRSEC DMR-0520404).
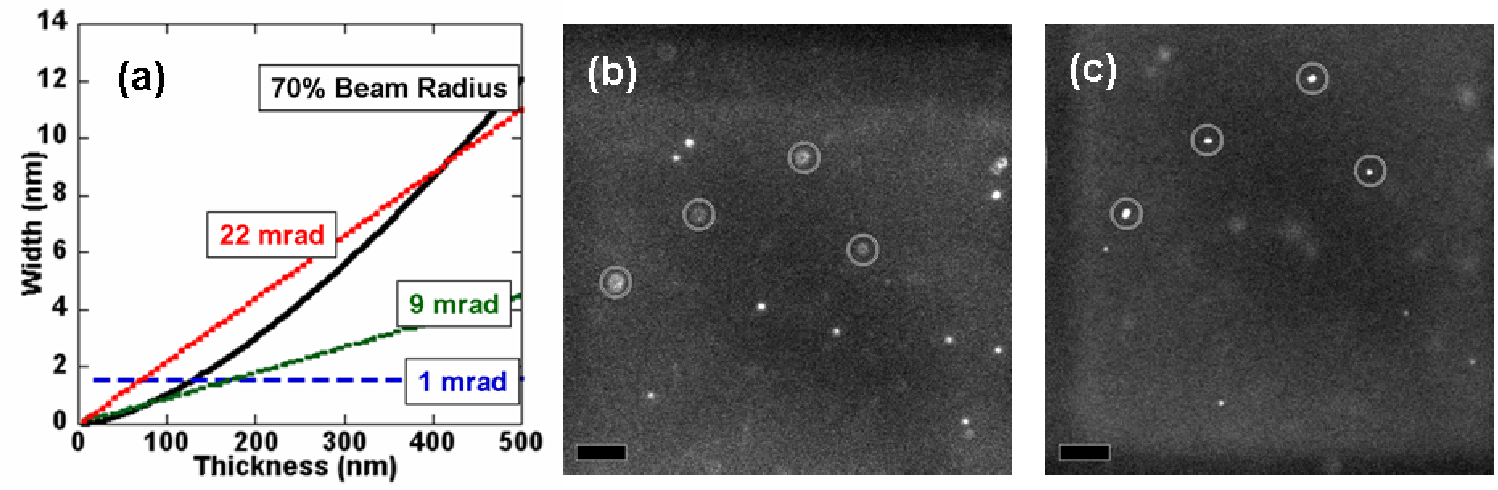

FIG. 1. (a) Beam spreading for $70 \%$ beam radius in amorphous carbon and beam divergence for 1, 9 and $22 \mathrm{mrad}$ apertures as a function of thickness. Beam divergence can be deduced from the size of the (b) top particles indicated by circles while beam is focused on the bottom. (c) Measurement of the blur of the bottom particles while beam is focused on the top confirms beam spreading and divergence measurements. Scale bars mark $20 \mathrm{~nm}$.
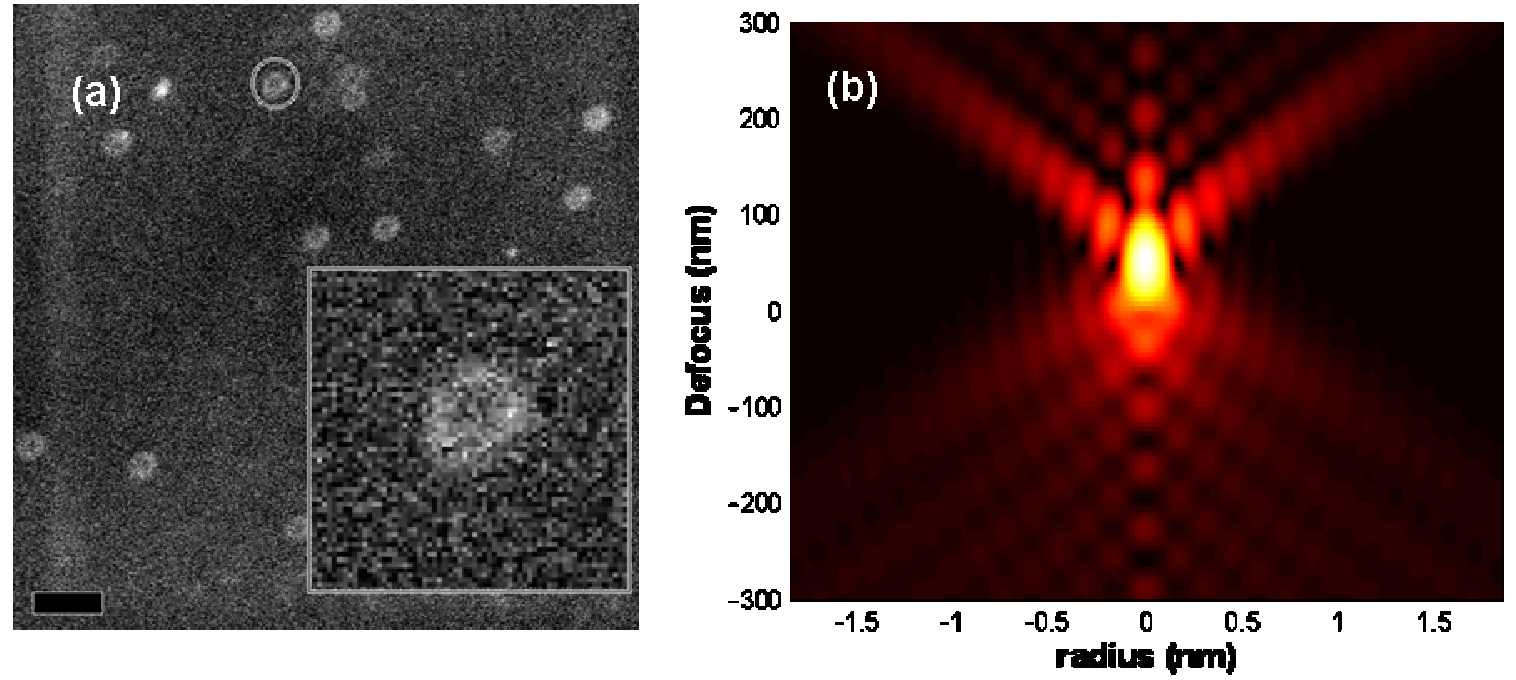

FIG. 2. (a) The top particles blur into donut-like shapes as the bottom particles come into focus in a $200 \mathrm{~nm}$ thick substrate when focused on the bottom. Scale bar is set at $20 \mathrm{~nm}$. (b) The point spread function for a series of defocus settings for $9 \mathrm{mrad}, \mathrm{C}_{\mathrm{s}}=1.2 \mathrm{~mm}$ and 200 $\mathrm{keV}$. The probe profile splits into two or more peaks above $50 \mathrm{~nm}$ defocus responsible for the donut shape blurring. 